\title{
Tecnologias de ensino utilizadas na Educação na pandemia COVID-19: uma revisão
}

\section{integrativa}

\author{
Teaching technologies used in Education in the COVID-19 pandemic: an integrative review \\ Tecnologías de enseñanza utilizadas en Educación en pandemia COVID-19: una revisión \\ integrativa
}

Recebido: 14/01/2021 | Revisado: 17/01/2021 | Aceito: 22/01/2021 | Publicado: 28/01/2021

\author{
Josaphat Soares Neto \\ ORCID: https://orcid.org/0000-0002-7077-0487 \\ Universidade Federa do Ceará, Brasil \\ E-mail: josasoaresneto@gmail.com \\ Francisco Victor Alves de Pinho \\ ORCID: https://orcid.org/0000-0002-1917-4524 \\ Universidade Federa do Ceará, Brasil \\ E-mail: victor.alvesp@alu.ufc.br \\ Heliene Linhares Matos \\ ORCID: https://orcid.org/0000-0003-3797-3124 \\ Universidade Federal do Ceará, Brasil \\ E-mail: hlmatos@unifor.br \\ Allyson Rodrigo de Oliveira Lopes \\ ORCID: https://orcid.org/0000-0003-3079-8634 \\ Universidade Federa do Ceará, Brasil \\ E-mail: allysonlopes85@gmail.com \\ Gilberto Santos Cerqueira \\ ORCID: https://orcid.org/0000-0001-6713-3772 \\ Universidade Federal do Ceará, Brasil \\ E-mail: giufarmácia@ @otmail.com \\ Emmanuel Prata de Souza \\ ORCID: https://orcid.org/0000-0001-9591-2202 \\ Universidade Federal do Ceará, Brasil
}

\begin{abstract}
Resumo
A pandemia de coronavírus (COVID-19) provocou a paralisação do ensino presencial, acarretando uma mudança imediata no processo educacional em todo o mundo. O objetivo deste estudo consiste em analisar por meio de uma revisão integrativa o uso das diversas tecnologias aplicadas no ensino básico e superior em diferentes áreas de conhecimento, durante a pandemia do COVID-19. A pesquisa é de natureza qualitativa sobre a relação e aplicação das tecnologias no/para o ensino. De acordo com o objetivo possui caráter descritivo e exploratório, utilizando como procedimentos técnicos uma revisão integrativa em relação à temática abordada. A pesquisa foi realizada por meio de artigos indexados nas bases de dados eletrônicos na Science Direct e Google Scholar, publicados em português e inglês, de 2010 a 2020, considerando os seguintes descritores: Tecnologias; Educação; Pandemia e COVID-19. Utilizou-se como critério de inclusão a coerência com o tema, e de exclusão a repetição de artigos ou não adequação ao tema. Foram encontrados 56 artigos $(n=56)$, que foram reduzidos para $(n=12)$ após análise. Os dados relatam que a inclusão das tecnologias, em especial as digitais incorporadas por meio de plataformas ao ensino remoto, possibilitam uma melhor qualificação no ensino, além de facilitar a aprendizagem os conteúdos desenvolvidos.
\end{abstract}

Palavras-chave: Tecnologias; Educação; Ensino; Pandemia; COVID-19.

\begin{abstract}
The coronavirus pandemic (COVID - 19) caused the on-campus teaching to be paralyzed, causing an immediate change in the educational process worldwide. The aim of this study is to analyze through an integrative review the use of the various technologies applied in teaching in different areas of knowledge, during the COVID-19 pandemic. The research is of a qualitative nature on the relationship and application of technologies in / for teaching. According to the objective, it has a descriptive and exploratory character, using as technical procedures an integrative review in relation to the theme addressed. The research was carried out through articles indexed in the electronic databases in Science Direct and Google Scholar, published in Portuguese and English, from 2010 to 2020, considering the following descriptors: Technologies; Education; Pandemia and COVID-19. Inclusion criteria were consistency with the theme, and exclusion was repetition of articles or non-adequacy to the theme. 56 articles $(n=56)$ were found, which were reduced to $(n=12)$ after analysis. The data report that the inclusion of technologies, especially the digital
\end{abstract}


ones incorporated by means of platforms for remote education, enables a better qualification in teaching, in addition to facilitating the learning of the developed contents.

Keywords: Technologies; Education; Teaching; Pandemic; COVID-19.

\section{Resumen}

La pandemia del coronavirus (COVID - 19) provocó la paralización de la enseñanza en el campus, provocando un cambio inmediato en el proceso educativo a nivel mundial. El objetivo de este estudio es analizar, a través de una revisión integradora, el uso de las distintas tecnologías aplicadas en la educación básica y superior en diferentes áreas del conocimiento, durante la pandemia COVID-19. La investigación es de carácter cualitativo sobre la relación y aplicación de tecnologías en / para la docencia. Según el objetivo, tiene un carácter descriptivo y exploratorio, utilizando como procedimientos técnicos una revisión integradora en relación al tema abordado. La investigación se realizó a través de artículos indexados en las bases de datos electrónicas en Science Direct y Google Scholar, publicados en portugués e inglés, de 2010 a 2020, considerando los siguientes descriptores: Tecnologías; Educación; Pandemia y COVID-19. Los criterios de inclusión fueron la coherencia con el tema y la exclusión fue la repetición de artículos o la no adecuación al tema. Se encontraron 56 artículos $(n=56)$, que se redujeron a $(n=12)$ después del análisis. Los datos informan que la inclusión de tecnologías, especialmente digitales incorporadas mediante plataformas de educación a distancia, permite una mejor calificación en la docencia, además de facilitar el aprendizaje de los contenidos desarrollados.

Palabras clave: Tecnologías; Educación; Ensenãnza; Pandemia; COVID-19.

\section{Introdução}

Dado o início do ano de 2020 o mundo foi surpreendido com o novo coronavírus SARS - CoV - 2, com isso afetou diretamente a educação e que mesmo em tempos difíceis não pode parar. O cotidiano da sociedade vem sendo transformado pela rápida evolução da tecnologia, provocando adaptações em diversas áreas de atuação, como as empresas, instituições governamentais e as instituições de ensino. As tecnologias compreendem os métodos ou técnicas criadas para o desenvolvimento da sociedade na tentativa de melhoria de vida (Ramos \& Campos, 2020).

É necessário reflexão e reavaliação da prática educacional dos envolvidos nos cursos de graduação, proporcionando alternativas para adentrar a realidade social, ética e crítica de um profissional de saúde nos processos de ensino e de aprendizagem. Em razão disso, são crescentes os desafios das instituições de ensino superior para formar profissionais com perfil crítico-reflexivo e inovador frente aos problemas do cotidiano (Bogdan \& Biklen, 1999).

A metodologia ativa vem se destacando nesse processo, a utilização de metodologias ativas no contexto educacional faz com que o aluno seja o ser central no processo de aquisição de conhecimento, saindo do papel de espectador na sala de aula (Diesel; Baldez; \& Martins, 2017).

Berbel (2011) acrescenta ainda que, as metodologias ativas têm como objetivo fazer com que seja desenvolvido o método de aprender, utilizando experiências reais ou simuladas, proporcionando ao aluno uma participação direta na construção da sua própria aprendizagem. Com isso, diversos métodos de ensino focados na metodologia ativa vêm sendo desenvolvidas remotamente com a utilização da gamificação.

Surge à necessidade de implementação de novas metodologias de ensino, como a inclusão das tecnologias digitais, associadas ao ensino tradicional nas instituições de Ensino Superior (IES). O conhecimento deve estar sempre acessível, de modo que o aprender se torne prazeroso (Fornaziero et al., 2010).

A transmissão de informações, de forma simples e cada vez mais acessível às pessoas pode influenciar na educação como um todo, emergindo a necessidade de aprendizado e adaptações no uso de novas tecnologias para facilitar o aprendizado e aumentar o interesse pelo conhecimento por parte dos discentes (Fornaziero \& Gil, 2003).

O Google Classroom é uma plataforma que está disponível desde 2014 e que se tornou uma das principais plataformas de acompanhamento de atividades utilizadas por professores de diversas áreas e redes durante esse período pandêmico, a inserção de vídeos do Youtube, postagens de atividades por professores e entrega das mesmas pelos alunos, 
materiais, links e todo acompanhamento de cada estudante cadastrados na turma como inserção de notas, são as principais funções da plataforma.

Embora longe do convencional, tem havido muito interesse no potencial dos aplicativos de Realidade Virtual (RV) para aprendizado e ensino nos últimos anos. A interatividade inerente às simulações permite que os alunos vejam resultados imediatos conforme criar modelos ou experimentar suas teorias sobre os conceitos modelados. A RV é popular entre os jovens e ocasionalmente tendem a ser usada na educação formal, como alguns jogos, por exemplo, (Mavrikios et al. 2019).

Surgiram avanços no sistema operacional computacional, como software de imagens e sistemas de imagens 3D que possibilitaram a construção de vídeos e modelos interativos a partir de imagens obtidas por modernas tecnologias, como tomografia computadorizada de raios X (TC), ressonância magnética (MRI) e tomografia de projeção óptica (OPT) (Lauridsen et al., 2011).

A tecnologia holográfica diferencia-se das tecnologias RV e Realidade Aumentada (RA), pois o holograma pode ser visualizado por muitos observadores ao mesmo tempo, sem a necessidade de usar propósitos especiais. A abordagem permitida pelos hologramas visa o uso muito mais amplo de novos métodos de aprendizagem, integrando a indústria e academia através da implantação de tecnologias holográficas (Mavrikios et al., 2019).

A utilização das simulações em ambiente virtual, nos mais variados cenários de educação e formação, não é recente. Inúmeras áreas de formação utilizam desde os simuladores de voo a simuladores de condução às intervenções cirúrgicas, como apoio ao ensino e à aprendizagem. São ferramentas, que há muito são estudadas por diversos trabalhos na busca de se analisar os efeitos cognitivos, comportamentais ou outros sobre as práticas pedagógicas (Morgado, 2020).

Outra ferramenta válida são os jogos digitais. Inúmeras pesquisas em educação buscam solucionar o potencial dos jogos eletrônicos no processo de ensino e aprendizagem em várias áreas do conhecimento. No campo educacional, nos últimos anos, as tecnologias da informação e da comunicação (TIC), alcançaram maior destaque e importância por refletirem maiores mudanças no modo de produzir, comunicar e sobre as formas de aprendizado (Ramos \& Campos, 2020).

A utilização do Kahoot na educação pode despertar novas experiências aos alunos, uma vez que para muitos é uma nova metodologia de aprendizagem, como também ampliar as perspectivas dos mesmos quanto a utilização do celular e computador para o desenvolvimento de uma aprendizagem de qualidade (Martins \& Gouveia, 2019).

Borba et al. (2020) destacam a utilização do kahoot durante o período pandêmico por professores da rede municipal, estadual, federal e privada, e constata-se a utilização por parte desses professores em todas as redes e principalmente por professores do Ensino Médio e pré-vestibulares.

As competições baseadas em jogos comprovam um processo de aprendizado desenvolvido nos alunos durante articulações em grupo, onde os mesmos pensam, discutem e resolvem problemas em conjunto como se fosse individualmente, enfatizando os benefícios de se trabalhar em equipe de forma colaborativa (Gravatá, 2013).

O desenvolvimento de estratégias educacionais gamificadas por professores de diversas áreas (como biologia, história, pedagogia, matemática, artes e letras) é observado que independente da disciplina é possível inserir os jogos digitais em determinados conteúdos. A interdisciplinaridade que o uso de jogos pode ajudar a promover envolvendo várias disciplinas é um fator importante que vale a pena ser discutido e avaliado (Alves; Minho \& Diniz, 2014).

São encontrados diversos benefícios com a utilização da gamificação na educação, porém, alguns limites devem ser levados em consideração. Para um desenvolvimento e uma boa aplicação da gamificação, deve-se atender às expectativas dos estudantes, evitando interpretações sobre essa estratégia que a considerem como uma metodologia superficial, desnecessária ou aplicada em excesso dentro do contexto escolar (Vieira et al.,. 2018).

É notável a relação, por parte da maioria dos alunos, com a tecnologia. Em vista disso, na preparação dos estudantes para o futuro, os modelos tradicionais não adquirem respostas diante das novas demandas (Prensky, 2012). Dessa forma, é 
necessário priorizar o desenvolvimento de habilidades e atitudes condizentes com o atual contexto da educação (Vieira; Tenreiro-Vieira \& Martins, 2011).

Inserir uma nova metodologia muitas vezes se torna desafiador, sobretudo quando está relacionada à educação, de modo que essa dificuldade é natural e diante da necessidade de mudanças no ensino tradicional, é inevitável a adição de novas tecnologias de ensino-aprendizagem. As dificuldades impulsionam o desenvolvimento da pesquisa sob a ótica das tecnologias digitais, especificamente, com o uso de holograma 3D e jogos digitais.

Nesse estudo de revisão integrativa, refletimos sobre a seguinte questão norteadora: Quais as principais tecnologias digitais inseridas no ensino com o surgimento da pandemia do COVID-19 no mundo? Nessa perspectiva, o objetivo da pesquisa é analisar por meio da revisão integrativa o uso das diversas tecnologias aplicadas no ensino de diferentes áreas de conhecimento, durante a pandemia do COVID-19.

\section{Metodologia}

O presente estudo trata-se de uma revisão integrativa sobre a temática: Tecnologias de ensino utilizadas na educação na pandemia do COVID-19. Conceitualmente a revisão integrativa refere-se a um estudo bibliográfico, de natureza qualitativa em que o mais importante é a interpretação por parte do pesquisador com suas opiniões sobre o fenômeno em estudo (Pereira et al,, 2018) e com técnicas e etapas pré-definidas, sujeitas a reprodução Rother (2007) e análise de informações científicas, com vista a identificar omissões de conhecimento, apontar estudos já produzidos e indicar prioridades para futuros estudos e/ou tomada de decisões (Paiva, et al., 2016).

Conforme Botelho, De Almeida e Macedo (2011), a revisão integrativa é constituída de seis fases, assim mencionadas: identificação do tema e a pergunta guia da pesquisa, escolha de critérios de inclusão e exclusão, identificação dos estudos pré-selecionados e selecionados, categorização destes estudos, análise e interpretação dos resultados e a apresentação da síntese/revisão do conhecimento, como mostra a (Figura 1) abaixo:

Figura 1: Etapas da revisão integrativa

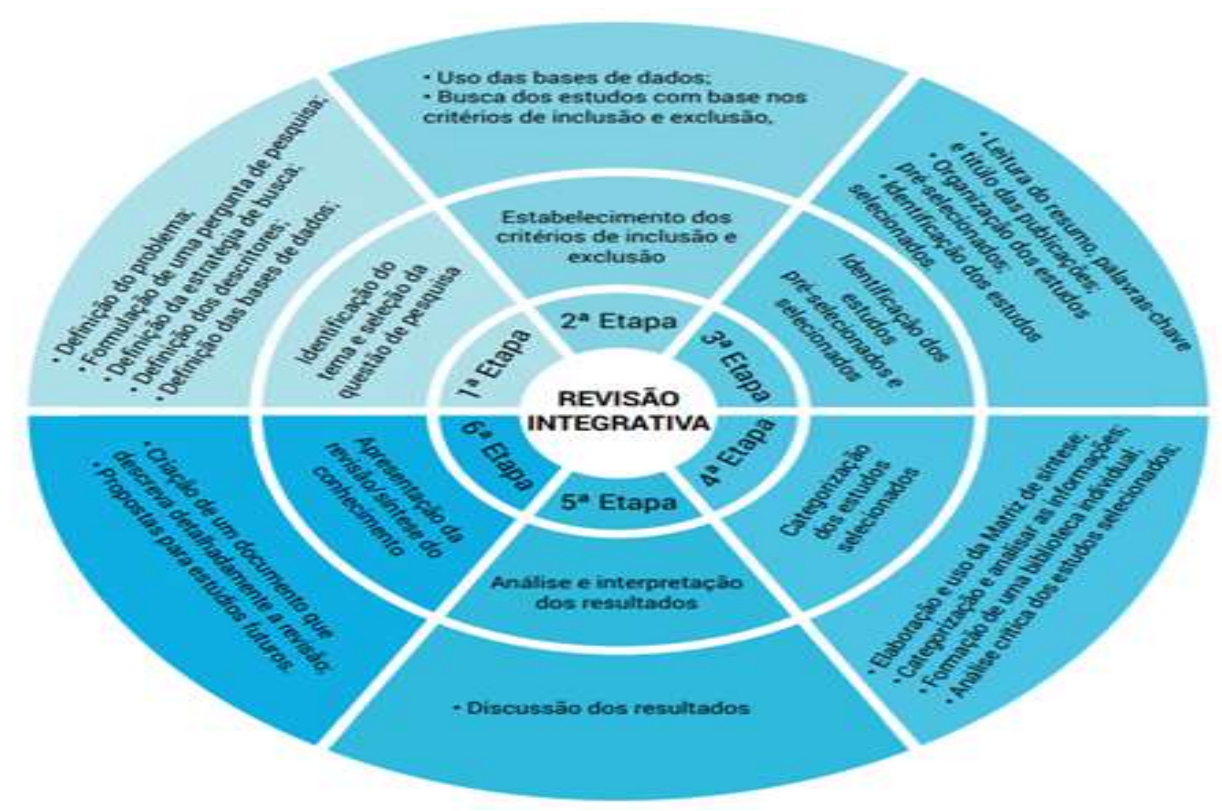

Fonte:

(Botelho; Cunha, Macedo, 2011, p. 129) 
De acordo com Botelho et al. (2011), o esquema mostra de forma detalhada as etapas de uma revisão integrativa, onde tem-se: $1{ }^{a}$ Etapa - A definição do problema, a formulação da pergunta norteadora da pesquisa, critérios para a busca e seleção de dados e definição das bases de dados; $2{ }^{\text {a }}$ Etapa - O uso das bases de busca selecionadas conforme os critérios atribuídos na seleção anteriormente; $3{ }^{\text {a }}$ Etapa - A leitura dos resumos e palavras chaves dos artigos na base de dados, afim de organizá-los para a seleção dos estudos; $4{ }^{\text {a }}$ Etapa - A categorização e análise dos estudos selecionados; 5 a Etapa - A análise e a discussão dos resultados e por último a $6{ }^{\text {a }}$ Etapa - Elaboração da tabela de revisão integrativa detalhando de forma clara e objetiva os dados mais relevantes encontrados na seleção dos artigos e na proposição de propostas para pesquisas futuras

Diante da definição da pergunta norteadora para a pesquisa e em consonância com as fases apontadas por Botelho, De Almeida \& Macedo (2011), os parâmetros de busca, seleção e análise dos trabalhos se deram inicialmente pela: i) seleção de artigos indexados nas bases de dados eletrônicas Science Direct e Google Scholar, publicados em português e inglês, de 2010 a 2020. ; ii) recorte temporal de 10 anos para a realização da pesquisa, ou seja, de 2010 a 2020; iii) uso dos termos "Tecnologia"; "Educação"; "Ensino"; "Pandemia" e "COVID-19' no título; iv) que contemplem o uso de diversos tipos de tecnologias ao ensino no que tange às suas implicações e desafios para um ensino significativo e de qualidade

A revisão foi restrita a artigos que abordam o uso de tecnologias aplicadas especialmente ao ensino superior de diversas áreas do conhecimento.

Estudos de revisão, artigos com duplicidade de dados; títulos e / ou resumos que não atendem aos critérios de inclusão foram excluídos, bem como trabalhos com ausência de informações pertinentes, totalizando 12 artigos para análise nesta revisão.

Assim, para a categorização deste estudo sobre o uso das tecnologias no ensino durante o isolamento social causado pela pandemia foram empregados vários critérios de seleção de artigos para que pudéssemos detalhar com maior profundidade a temática e suas particularidades.

Foram consideradas elegíveis as publicações compreendidas no intervalo de tempo entre o dia 1 de janeiro de 2010 e o dia 10 de outubro de 2020, momento em que foi realizada a pesquisa. Não foi definida qualquer restrição quanto ao tipo de estudo.

De um total de 286 documentos identificados, (06) foram excluídos por se encontrarem duplicados. Seguidamente, iniciou-se o processo de seleção dos artigos com a aplicação de testes de triagem. Inicialmente foi aplicado a (280) estudos. Após a análise do título e resumo de cada um dos artigos candidatos à exclusão nesta etapa da triagem foi possível excluir (224) artigos. Restando (56) artigos elegíveis foi feita uma leitura integral de cada um dos artigos e na análise dos respectivos critérios previamente definidos para esta revisão permitiu que (40) artigos fossem excluídos. Os artigos incluídos na síntese qualitativa a serem trabalhados em nossa temática ficaram representados em número de (12) artigos.

Dos motivos de exclusão, destaca-se: o objetivo do estudo é diferente da temática desta revisão (16), a problemática inserida no texto não se refere ao contexto da revisão (14), o estudo não cumpre critérios de inclusão (04), e o sujeito do estudo é diferente do sujeito da revisão (10).

Na Figura 2 tem-se a representação do processo seletivo dos estudos por meio Prisma 2009 Flow Diagram, tendo como Palavras chaves: “Tecnologias"; "Educação";"Ensino"; "Pandemia” e "COVID-19"

Após a seleção criteriosa dos 12 artigos a serem incluídos para uma análise qualitativa, elaboramos uma tabela onde mostra os seguintes dados: Nome dos autores, revista/ data de publicação, os objetivos, o desenho do estudo, resultados, desfecho. 
Figura 2: Representação do processo seletivo dos estudos por meio Prisma 2009 Flow Diagram.
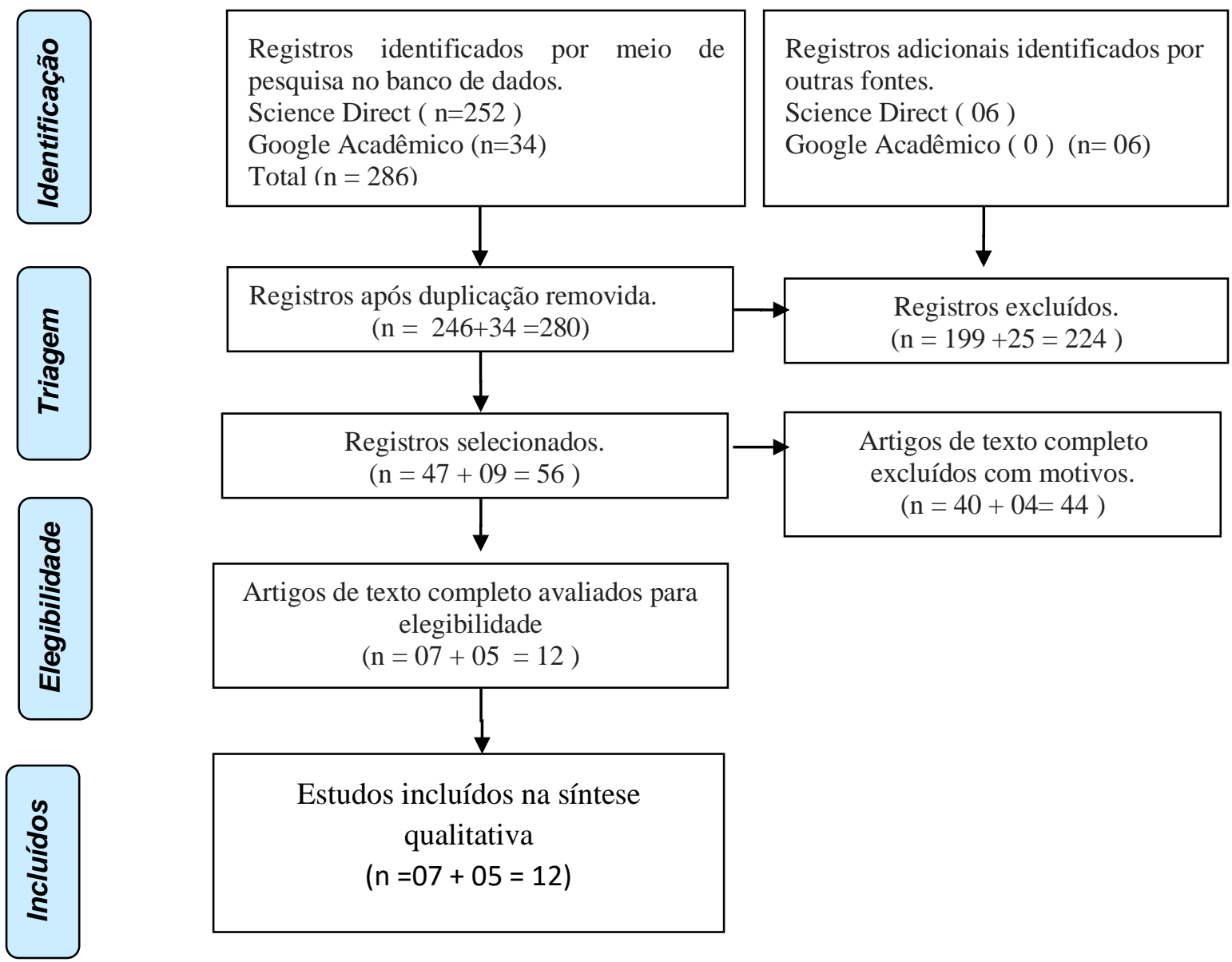

Artigos de texto completo avaliados para elegibilidade

$$
(\mathrm{n}=07+05=12)
$$

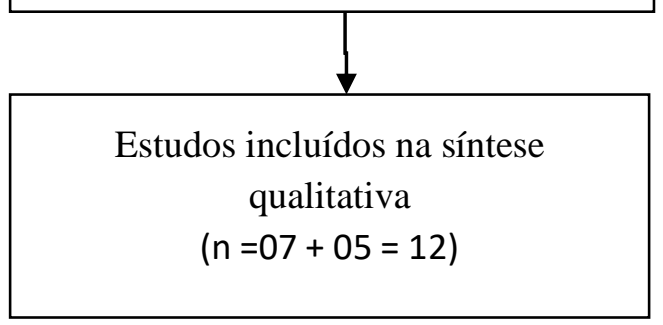

Fonte: Elaborado pelo autor no Prisma 2009 Flow Diagram (2020).

\section{Resultados e Discussão}

O estudo da temática em questão teve como foco principal analisar por meio de uma revisão integrativa o uso das diversas tecnologias aplicadas no ensino em diferentes áreas de conhecimento, durante a pandemia do COVID-19, buscando identificar os impactos causados por essa integração tecnológica na transposição didática dos conteúdos aos alunos, refletindo na aprendizagem dos mesmos e na qualificação da prática docente. Os resultados da revisão integrativa obtidos no estudo foram analisados e colocados de forma explicativa em uma tabela representada no Quadro 1, onde se tem detalhado os autores e o ano de publicação do artigo selecionado, a revista da publicação, o objetivo principal da pesquisa desenvolvida no artigo, o desenho do estudo da pesquisa, os resultados encontrados e o desfecho com as considerações finais. 
Quadro-1: Tabela Revisão Integrativa.

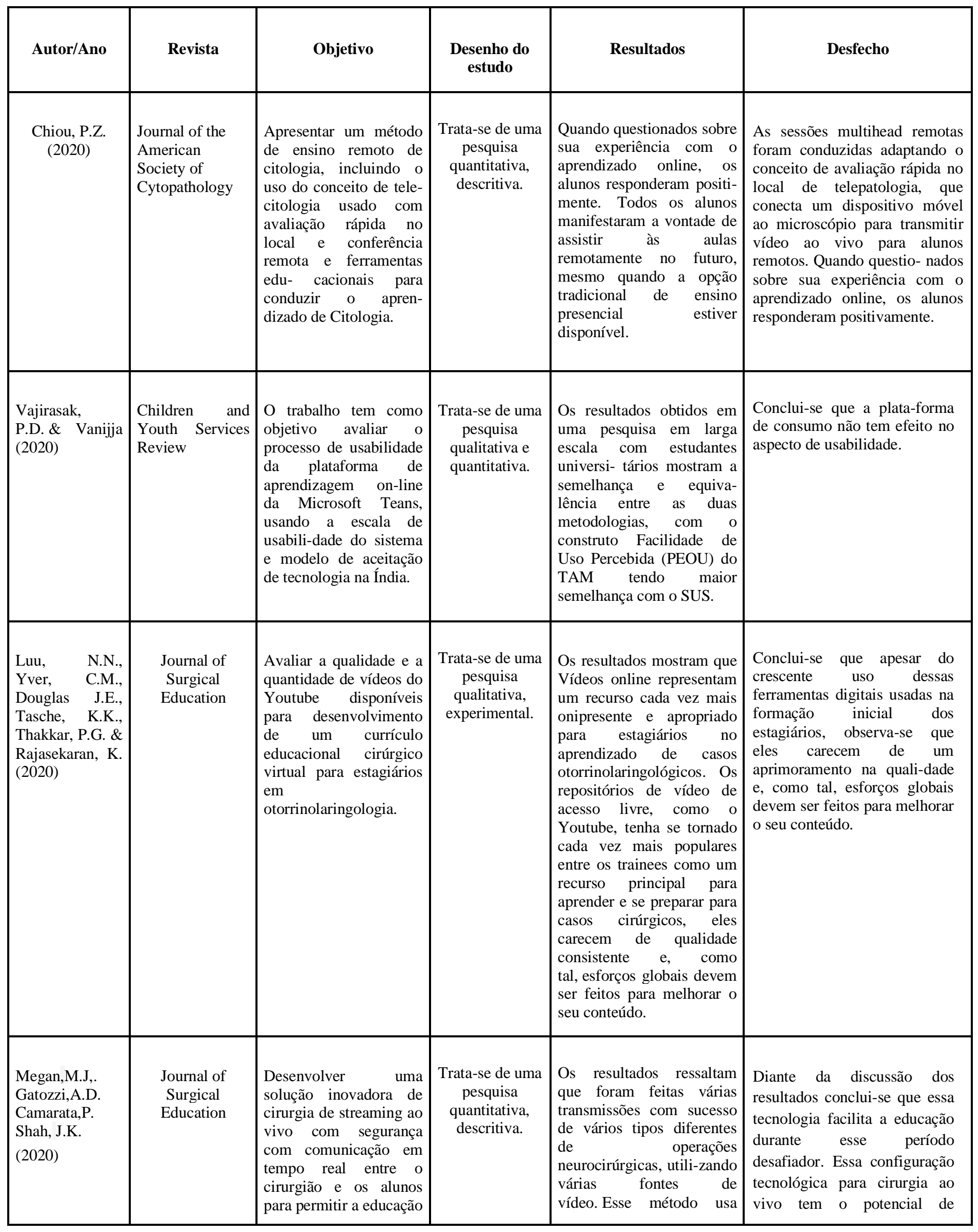




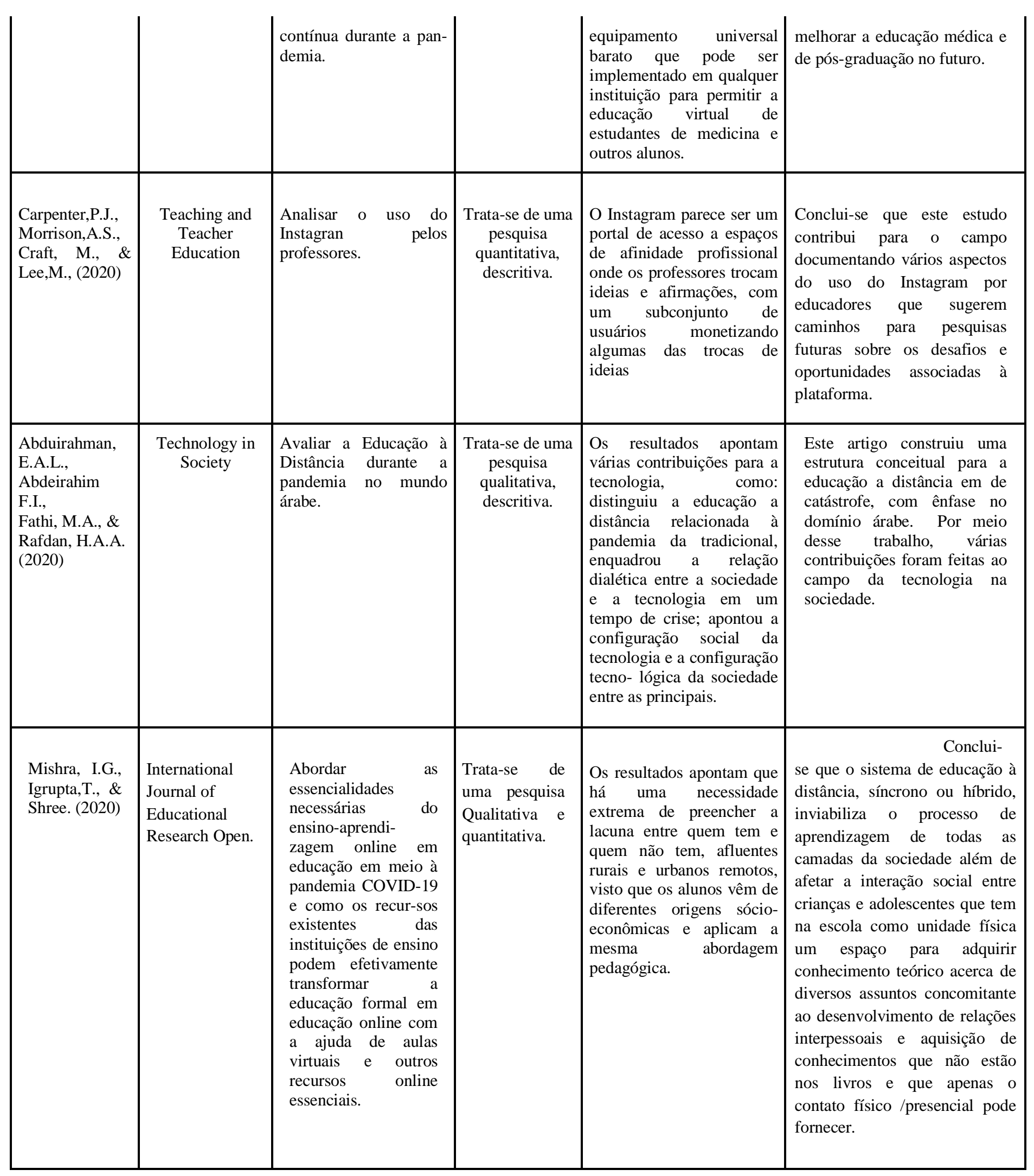




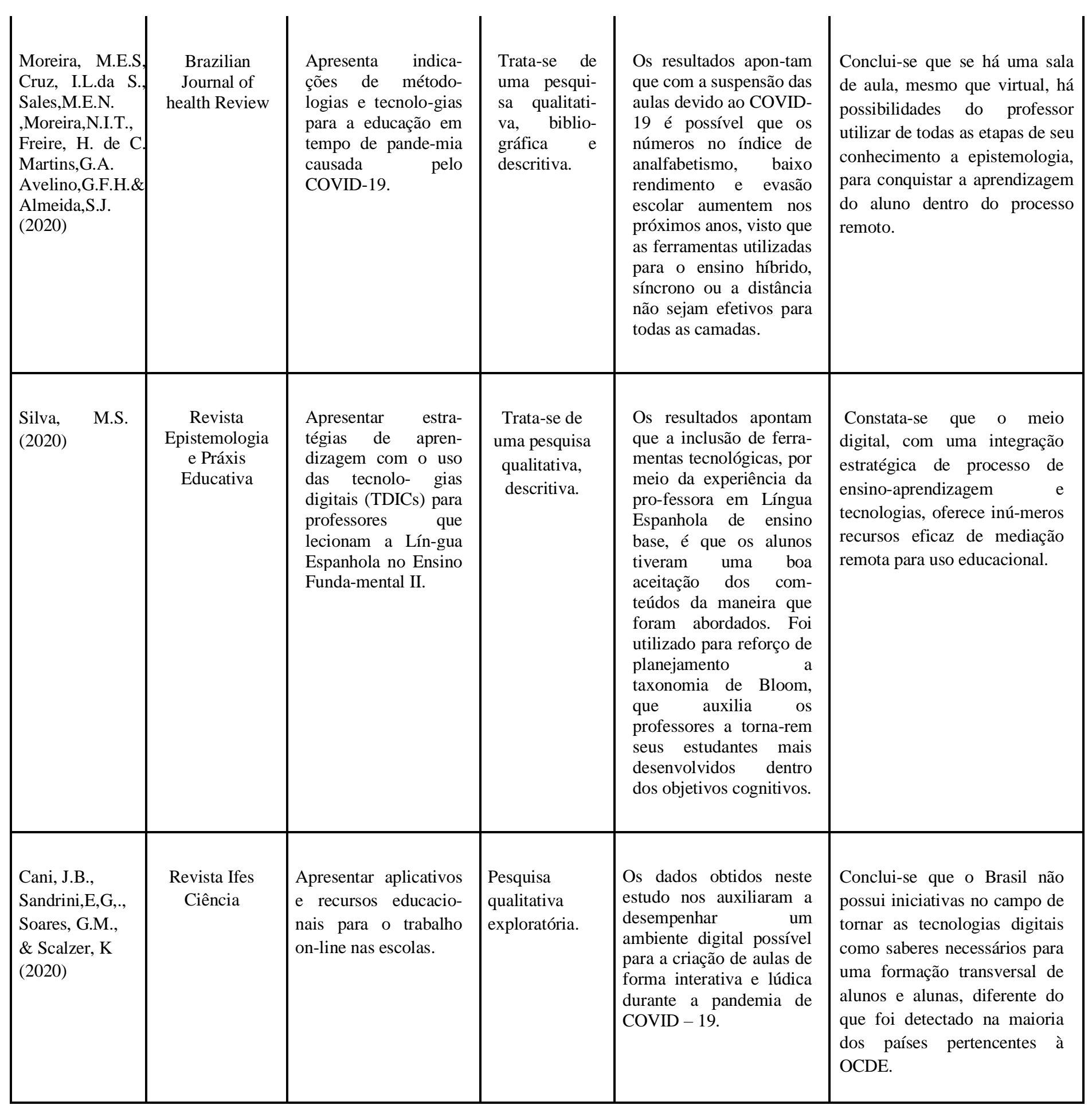




\begin{tabular}{|c|c|c|c|c|c|}
\hline $\begin{array}{l}\text { Arruda, E.P. } \\
(2020)\end{array}$ & Uni Rede & $\begin{array}{l}\text { Evidenciar } \\
\text { excepcionalidade da } \\
\text { situação que levou } \\
\text { inúmeros países a } \\
\text { desen- volver ações } \\
\text { de educação remota } \\
\text { emergencial e as } \\
\text { implicações nos } \\
\text { diferentes níveis } \\
\text { educacionais. }\end{array}$ & $\begin{array}{c}\text { Trata-se de uma } \\
\text { pesquisa } \\
\text { qualitativa, } \\
\text { bibliográfica. }\end{array}$ & $\begin{array}{l}\text { Temos observado uma } \\
\text { amplificação } \\
\text { resistências de } \\
\text { implementação } \\
\text { educação da } \\
\text { emergencial na educação } \\
\text { brasileira, em seus } \\
\text { diferentes níseis, } \\
\text { conforme observado na } \\
\text { leitura de documentos } \\
\text { sindicais e de atas e } \\
\text { resoluções de Conselhos } \\
\text { Universi-tários de } \\
\text { universidades públicas. } \\
\text { A maior parte dos } \\
\text { argumentos envolve a } \\
\text { dimensão dis } \\
\text { disponibilidade de } \\
\text { equipamentos para todos, } \\
\text { ou seja, a equidade. }\end{array}$ & $\begin{array}{l}\text { No ensino da enfermagem, as } \\
\text { discussões relacionadas ao uso } \\
\text { de tecnologias remotas em sala } \\
\text { de aula sempre foi um ponto } \\
\text { de debate. Entretanto, com a } \\
\text { necessidade da inclusão dessas } \\
\text { ferramentas para a } \\
\text { continuidade de aulas no } \\
\text { formato não presencial } \\
\text { decorrente da estratégia do } \\
\text { isolamento social motivada } \\
\text { pela pandemia do COVID-19, } \\
\text { pode oportunizar a se ter um } \\
\text { novo olhar sobre o assunto e } \\
\text { que talvez com esse momento } \\
\text { vivido se haja oportunidade de } \\
\text { ampliar o debate sobre uso } \\
\text { dessas metodologias remotas } \\
\text { no ensino em saúde, buscando } \\
\text { uma reflexão sobre a interação } \\
\text { destas com os demais métodos } \\
\text { de ensino já implementados. }\end{array}$ \\
\hline $\begin{array}{l}\text { Bezerra, } \\
\text { I.M.P. (2020) }\end{array}$ & $\begin{array}{c}\text { J Hum Growth } \\
\text { Dev }\end{array}$ & $\begin{array}{l}\text { Descrever o estado da } \\
\text { arte sobre o ensino de } \\
\text { Enfermagem e os } \\
\text { desafios do uso de } \\
\text { tecnologias remo- tas } \\
\text { em época de pandemia } \\
\text { da Corona vírus. }\end{array}$ & $\begin{array}{l}\text { Pesquisa } \\
\text { qualitativa, } \\
\text { descritiva, } \\
\text { experimental }\end{array}$ & $\begin{array}{l}\text { Evidencia-se que } \\
\text { vivenciar os efeitos da } \\
\text { pandemia de corona vírus } \\
\text { (COVID-19) no setor } \\
\text { educacional na formação } \\
\text { em saúde, em especial no } \\
\text { campo da enfermagem, } \\
\text { vai além de uma } \\
\text { reorganização estrutural } \\
\text { dos cursos, pois implica } \\
\text { em mudança atitudinal } \\
\text { dos gestores, docentes e } \\
\text { discentes para que } \\
\text { reformulem as práticas de } \\
\text { ensino. }\end{array}$ & $\begin{array}{l}\text { Conclui-se que desenvolver } \\
\text { abordagens multimodais para } \\
\text { atingir os objetivos do conteúdo } \\
\text { do curso para um melhor } \\
\text { resultado de aprendizagem pode } \\
\text { ser uma ideia melhor para lidar } \\
\text { com a complexidade da } \\
\text { educação online. }\end{array}$ \\
\hline
\end{tabular}

Fonte: Elaborado pelo autor (2020)

\section{Considerações Finais}

O Quadro 1 acima descrito, sintetiza os dados que foram achados e posteriormente analisados nos 12 artigos selecionados sobre a temática: Tecnologias de ensino utilizadas na educação na pandemia COVID-19, obedecendo os critérios previstos nas plataformas digitais da Science Direct e Google Acadêmico na pesquisa realizada.

Ao longo da pesquisa conseguimos selecionar alguns resultados relevantes inseridos nos artigos que trabalham com a temática: Tecnologias de ensino utilizadas na educação na pandemia COVID-19 e observamos de maneira ampla que as ferramentas tecnológicas inseridas no processo educacional e profissional durante este período emergente trouxe várias reflexões sobre o uso dessa tecnologia pela sociedade nos mais diversos segmentos.

Inicialmente exploramos as discussões sobre os resultados achados do uso das tecnologias no ensino superior, em especial, na área da saúde, envolvendo os cursos de graduação, pós-graduação, e posteriormente chegando ao aprimoramento 
dos profissionais desta área. Em seguida, discutimos a presença das tecnologias no ensino básico, relacionando o ensino remoto com a modalidade de ensino presencial.

Em relação ao Ensino Básico, (Chiou, 2020) propôs um método de ensino remoto de citologia, incluindo o uso do conceito de telecitologia usado com avaliação rápida no local e conferência remota e ferramentas educacionais para conduzir o aprendizado de Citologia. As sessões multi-head remotas, conduzidas pela adaptação do conceito ROSE de telecitologia com um dispositivo móvel acoplado ao microscópio, são uma abordagem eficaz para o ensino à distância em tempos de distanciamento social e é uma ferramenta promissora para o ensino de citologia do futuro.

Segundo Khurana (2012) o modelo expresso por telecitologia possibilita a discussão de alterações microscópicas que podem ser compartilhados com outros profissionais à distância. A imagem pode ser inserida na tela do computador ou do smartphone. Além disso, as lâminas coletadas podem ser arquivadas para a avaliação diagnóstica e tratamento de doenças diversas, como o câncer, e servir de base na geração de atlas digital para ser usado nos cursos de graduação e pós-graduação da área da saúde.

(Moreira et al., 2020), apresentou indicações de metodologias e tecnologias para a educação em tempo de pandemia pelo COVID-19. O autor faz reflexões críticas em relação ao sistema de educação à distância, síncrono ou híbrido, que inviabiliza o processo de aprendizagem de todas as camadas da sociedade além de afetar a interação social entre crianças e adolescentes. Além disso, afirma que haverá um aumento no índice de analfabetismo, no baixo rendimento e na evasão escolar nos próximos anos, visto que as ferramentas utilizadas para o ensino híbrido, síncrono ou a distância não são efetivos para todas as camadas.

Em relação às críticas que se observam em debates acadêmicos sobre o sistema de educação à distância e demais modalidades de ensino que envolve o ambiente virtual se fazem necessário levar em consideração que os processos de adaptações entre as práticas pedagógicas e o uso das tecnologias são de suma importância para que haja qualidade de construção do conhecimento, porém, precisam-se haver formações pontuais para a comunidade escolar (professores, alunos e gestores) para essa nova conjuntura que configura o cenário atual.

Segundo Mill (2012, p. 48), "no âmbito da EaD, antes do cadastramento dos alunos, uma disciplina precisa ser minuciosamente planejada para sua realização, sendo a docência permeada por uma espécie de pedagogia da previsão" e com práticas que promovam a interação entre professor-aluno nas bases digitais.

(Silva et al., 2020), apresentou estratégias de aprendizagem com o uso de tecnologias digitais para professores do Ensino Fundamental II que lecionam a Língua Espanhola. O autor afirma que se há uma sala de aula, mesmo que virtual, há possibilidades do professor utilizar de todas as etapas de seu conhecimento a epistemologia para conquistar a aprendizagem do aluno dentro do processo remoto.

(Cani et al., 2020), apresentou aplicativos e recursos educacionais para o trabalho on-line nas escolas básicas. A pesquisa foi desenvolvida relacionando a percepção dos professores com o uso das tecnologias digitais, incluindo também suas habilidades. $\mathrm{O}$ autor constatou que o meio digital, com uma integração estratégica de processo de ensino-aprendizagem e tecnologias, oferece inúmeros recursos eficazes de mediação remota para uso educacional.

Não basta apenas o educador mudar a sua prática educacional ou inserir ferramentas digitais, como aplicativos inseridos em smartphone para promover o interesse do aluno para aquele determinado conteúdo. $\mathrm{O}$ aluno também precisa pensar sobre o conteúdo, interagir com a ferramenta disponível, utilizando-o como suporte a fim de tirar suas próprias conclusões sobre o assunto abordado.

Nessa perspectiva, Moraes (1997) comenta:

“[...] a busca de melhoria da qualidade educacional significará o aperfeiçoamento do processo de aprendizagem, que, além de levar em conta as necessidades dos usuários em cada etapa de sua trajetória, busca também novas estratégias 
de aprendizagem mais adequadas à produção do conhecimento, cada vez mais atualizado, ampliado, que induz à expansão da cognição humana e à crescente intelectualização do trabalho". (Moraes 1997, p.7)

(Arruda, 2020), analisou a excepcionalidade da situação que levou inúmeros países, em especial, o Brasil a desenvolver ações de educação remota emergencial e as implicações nos diferentes níveis educacionais Os resultados apontam que o Brasil não possui iniciativas no campo de tornar as tecnologias digitais como saberes necessários para uma formação transversal de alunos e alunas, diferente do que foi detectado na maioria dos países pertencentes à OCDE.

Diante deste cenário é fundamental analisar a problemática por todos os ângulos e refletir sobre três questões, que se relacionam quando falamos em iniciativas do estado em tornar as tecnologias digitais como saberes necessários no processo educacional: o acesso à internet, a qualidade dos artefatos tecnológicos de alunos e professores, e o domínio e formação para o uso desses artefatos.

Conforme (Cani et al.,2020) ressalta:

“[...] cabe questionarmos não somente acerca do acesso às tecnologias, mas, sobretudo, da possibilidade de serem oferecidas a professores e alunos condições para uso pleno dos recursos tecnológicos, de modo a favorecer uma aprendizagem interativa e colaborativa. Sabemos que são muitos os desafios e os fatores implicados, desde a falta de estrutura tecnológica das escolas, formação dos próprios professores e alunos para um uso crítico das tecnologias". (Cani et al., 2020, p. 24).

(Mishra et al., 2020), abordou as essencialidades necessárias do ensino-aprendizagem online em educação em meio à pandemia COVID-19 e como os recursos existentes das instituições de ensino podem efetivamente transformar a educação formal em educação on line com a ajuda de aulas virtuais e outros recursos online essenciais. Nesse estudo, os resultados apontam que há uma necessidade extrema de preencher a lacuna entre “quem tem e quem não tem”, afluentes rurais e urbanos remotos, visto que os alunos vêm de diferentes origens socioeconômicas e aplicam a mesma abordagem pedagógica. A não compreensão das diferenças individuais dos alunos pode resultar em conclusões tendenciosas, especificamente porque o modo de ensino-aprendizagem on-line, que aqui é infantil, pode não atender ao seu objetivo final.

(Caperten et al., 2020), analisou o uso do Instagram pelos professores tanto da escola básica, como também do nível superior. Ficou constatado que o Instagram parece ser um portal de acesso a espaços de afinidade profissional onde os professores trocam ideias e afirmações, e sugerem caminhos para pesquisas futuras sobre os desafios e oportunidades associados à plataforma.

No Ensino superior, (Debajotti \& Vajirasak, 2020) avaliou com estudantes universitários o processo de usabilidade da plataforma de aprendizagem on line da Microsoft Teans, usando a escala de usabilidade do sistema e modelo de aceitação de tecnologia na Índia, onde concluiu que a plataforma de consumo não tem efeito no aspecto de usabilidade.

(Luu et al., 2020), avaliou a qualidade e a quantidade de vídeos do YouTube disponíveis para desenvolvimento de um currículo educacional cirúrgico virtual para estagiários em otorrinolaringologia. O autor identifica que apesar do crescente uso dessas ferramentas digitais usadas na formação inicial dos estagiários, observa-se que eles carecem de um aprimoramento na qualidade.

Apesar de grande parte dos vídeos da plataforma do YouTube apresentarem uma qualidade limitada em sua confecção e aprofundamento acadêmico, vale ressaltar que na interação das práticas pedagógicas com o uso do vídeo ocorre a aprendizagem significativa, pois o conhecimento sofre um processo de contínua elaboração e reelaboração de significados. (Moreira, 2006).

(Megan et al., 2020), desenvolveu uma solução inovadora de cirurgia de streaming ao vivo com segurança e com comunicação em tempo real entre o cirurgião e os alunos para permitir a educação contínua durante a pandemia. Os resultados mostram que essa configuração tecnológica para cirurgia ao vivo tem o potencial de melhorar a educação médica e de pósgraduação no futuro. 
(Abduirahman et al., 2020), avaliou. a Educação à Distância durante a pandemia no mundo árabe, onde, os resultados apontam várias contribuições para a tecnologia, tais como: distinguiu a educação a distância relacionada à pandemia da tradicional; enquadrou a relação dialética entre a sociedade e a tecnologia em um tempo de crise; apontou a configuração social da tecnologia e a configuração tecnológica da sociedade entre as principais.

(Bezerra, 2020), descreveu o estado da arte sobre o ensino de Enfermagem e os desafios do uso de tecnologias remotas em época de pandemia da Corona vírus. O autor ressalta, que no ensino da enfermagem, a discussão relacionada ao uso de tecnologias remotas em sala de aula sempre foi um ponto de debate, entretanto, com a necessidade da inclusão dessas ferramentas para a continuidade de aulas no formato não presencial devido ao isolamento social, pode possibilitar uma maior análise sobre o uso dessas metodologias remotas no ensino em saúde, buscando uma reflexão sobre a interação destas com os demais métodos de ensino já implementados.

Todos esses estudos são animadores e se tornam relevantes, pois possibilita uma maior reflexão sobre a viabilidade de forma mais efetiva do uso de novas tecnologias agregadas às metodologias que venham proporcionar uma melhoria na qualidade do ensino, no maior rendimento da aprendizagem dos conteúdos abordados, num maior aprimoramento profissional.

Assim, diante da análise dos resultados concordamos que este repensar perpassa pela integração dessas tecnologias ao fazer pedagógico, com vistas a uma formação que vise a uma nova ética e nova estética (Rojo, 2012), afinal de contas, vivemos uma era de mudanças frenéticas que demandam na educação capaz de tornar os alunos “[...] aprendizes autônomos, críticos, bem informados, cooperativos, colaborativos e que saibam usufruir plenamente, com segurança e com responsabilidade, das oportunidades que lhes são oferecidas nos ambientes digitais" (Coscarelli, 2018, p. 33-34)

\section{Agradecimentos}

Agradeço ao apoio da Universidade Federal do Ceará - UFC, do Departamento de Morfologia por meio do Prof. Dr. Gilberto Santos Cerqueira e Prof. Dr. e orientador Emmanuel Prata de Souza ambos professores do Curso de Pós-Graduação em doutorado em Ensino de Ciências Morfofuncionais da UFC e da Fundação Cearense de Apoio ao Desenvolvimento Científico e Tecnológico- Fucap-Ce pelo apoio financeiro a essa pesquisa.

\section{Referências}

Abduirahman, E. A. L., Abdeirahim F. I., Fathi, M. A., \& Rafdan, H. A. A. Distance education as a response to pandemics: Coronavirus and Arab culture, Technology in Society, Volume 63, November 2020,

Alves, L. R. G., Minho, M., \& Diniz, M. Gamificação: diálogos com a educação. In: Fadel, L. M., Ulbrich, V. R., Batista, R., \& Vazin, T. (Org.). Gamificação na educação: Pimenta Cultural, 1, 73-95, 2014.

Bogdan, R., \& Biklen, S. Investigação qualitativa em educação: uma introdução à teoria e métodos: Porto, 1999.

Borba, R. C. do N., Teixeira, P. P., Fernandes, K. de O. B., Bertagna, M., Valença, C. R., \& Souza, L. H. P. de. Percepções docentes e práticas de ensino de ciências e biologia na pandemia: uma investigação da Regional 2 da SBEnBio. Revista de Ensino de Biologia da SBEnBio, [S. 1.], 13(1), 153-171, 2020. 10.46667/renbio.v13i1.337.

Cani, J. B., Sandrini, E. G. C., Soares, G. M., \& Scalzer, K. Educação e covid-19: a arte de reinventar a escola mediando a aprendizagem "prioritariamente" pelas TDIC. Revista Ifes Ciência, v. 6, Edição Especial, n. 1, 2020, p. 23-39. https://ojs.ifes.edu.br/index.php/ric/article/download/713/484.

Coscarelli, C. V. Perspectivas culturais de uso de tecnologias digitais e a educação. Revista Brasileira de Alfabetização, Belo Horizonte-MG, 1(8), 33-56| jul./dez.2018. http://abalf.org.br/revistaeletronica/index.php/rabalf/article /view/293/211.

Debajyoti, P., \& Vajirasak, V. Perceived usability evaluation of Microsoft Teams as an online learningplatform during COVID-19 using system usability scale and technology acceptance model in India. Children and Youth Services Review. v.119.2020.

Diesel, A., Baldez, A. L. S., \& Martins, S. N. Os princípios das metodologias ativas de ensino: uma abordagem teórica. Revista Thema, $14(1), 268-288,2017$.

Gravata, A. et al. Volta ao mundo em 13 escolas: Fundação Telefônica: A. G., 2013.

Fornaziero, C. C., \& Gil, C. R. R. Novas tecnologias aplicadas ao ensino da anatomia humana. Revista Brasileira de Educação Médica, $27(2), 141-146,2003$. 
Research, Society and Development, v. 10, n. 1, e51710111974, 2021

(CC BY 4.0) | ISSN 2525-3409 | DOI: http://dx.doi.org/10.33448/rsd-v10i1.11974

Fornaziero, C. C., Gordan, P. A., Carvalho, M. A. V., Araujo, J. C., \& Aquino, J. C. B. O Ensino da Anatomia: Integração do Corpo Humano e Meio Ambiente. Revista Brasileira De Educação Médica, 34, (2), 290-297, 2010.

Khurana, K. K. Telecytology and its evolving role in cytopathology. Diagn Cytopathol. 2012 Jun; 40(6):498-502. 10.1002 / dc.22822.

Lauridsen H. et al. Inside out: modernas técnicas de imagem para revelar a anatomia animal. PLoS ONE 6, e17879. 2011. (10.1371 / journal.pone.0017879).

Martins, E. R., \& Gouveia, L. M. B. (2019). Aprendizagem Móvel com a Tecnologia Educacional Kahoot: uma discussão da perspectiva dos aprendizes. Revista EducaOnline, 13(3), 37-57.

Mavrikios, D. et al. Using Holograms for visualizing and interacting with educational content in a Teaching Factory. Procedia Manufacturing, 31, 404-410, 2019.

Mill, D. Docência virtual: uma visão crítica. (1. ed.). Editora Papirus, 2012

Moraes, M. C. Subsídios para Fundamentação do Programa Nacional de Informática na Educação: SEED/MEC, 1997.

Morgado, L. Os mundos virtuais e o ensino-aprendizagem de procedimentos. Revista Educação e cultura contemporânea, 6(13), 2020.

Moreira, M. A. (2006). A teoria da aprendizagem significativa e sua implementação em sala de aula. Brasília: Editora da UnB.

Paul, Z. C. Learning cytology in pandemic times: an institutional educations experience with distance learning. Journal of the American Society of Cytopathology. 9, 579 a 585, 2020.

Pereira, A. S., Shitsuka, D. M., Parreira, F. J., \& Shitsuka, R. (2018). Metodologia da pesquisa científica. Ed.UAB/NTE/UFSM. https://repositorio.ufsm.br/bitstream/handle/1/15824/Lic_Computacao_Metodologia_Pesquisa Cientifica.pdf?sequence=1

Prensky, M. Aprendizagem baseada em jogos digitais. [S1], 2012. Editora SENAC.

Ramos, D. K., Campos, T. R. O uso de jogos digitais no ensino de Ciências Naturais e Biologia: uma revisão sistemática de literatura. Revista Electrónica de Enseñanza de las Ciencias, 19(2), 450-473, 2020.

Rojo, R. Pedagogia dos multiletramentos: diversidade cultural e de linguagens na escola. In: Rojo, R.; Moura, E. (org.). Multiletramentos na escola: Parábola Editorial, 2012.

Silva, D. dos S., Andrade, L. A. P., \& Santos, S. M. P. dos. Teaching alternatives in pandemic times. Research, Society and Development, [S. l.], v. 9, n. 9, p. e424997177, 2020. 10.33448/rsd-v9i9.7177.

Vieira, R. M., Tenreiro-Vieira, C., \& Martins, I. P. Critical thinking: Conceptual clarification and its importance in science education. Science education international, 22(1), 43-54, 2011.

Vieira, A. de S. et al. O estado da arte das práticas de gamificação no processo de ensino e aprendizagem no ensino superior. Revista Brasileira de Ensino Superior, Passo Fundo, 4(1), 5-23, jan/mar., 2018 